\title{
A middle-aged lady with near-fatal respiratory failure
}

\author{
Pankti Sheth, Shibdas Chakrabarti, Neeraj Kumar Gupta, Pranav Ish, Rohit Kumar, Nitesh Gupta \\ Department of Pulmonary and Critical Care Medicine, Vardhman Mahavir Medical College and Safdarjung Hospital, \\ New Delhi, India
}

\begin{abstract}
Tuberculosis is an uncommon cause of acute hypoxemic respiratory failure. We present a case of a middle-aged woman with near-fatal respiratory failure due to underlying tuberculosis with favorable clinical recovery. Early diagnosis and treatment are imperative to prevent morbidity, mortality, and disease transmission.
\end{abstract}

Correspondence: Nitesh Gupta, Department of Pulmonary and Critical Care Medicine, Vardhman Mahavir Medical College \& Safdarjung Hospital, Room number 638, Superspeciality block, New Delhi 110029 , India.

E-mail: niteshgupta2107@gmail.com

Key words: ARDS; tuberculosis; respiratory failure.

Contributions: PS, SC, NG, conceived the study; PS, collected the data; SC, PI, data verification; PS, PI, NG, manuscript drafting. All the authors have read and approved the final version of the manuscript and agreed to be accountable for all aspects of the work.

Conflict of interest: The authors declare that they have no competing interests, and all authors confirm accuracy.

Ethics approval: No ethical committee approval was required for this Case Report by the Department, because this article does not contain any studies with human participants or animals.

Consent for publication: Written informed consent was taken from the patient for sharing clinical details and radiological images.

Received for publication: 27 September 2021.

Accepted for publication: 20 December 2021.

Publisher's note: All claims expressed in this article are solely those of the authors and do not necessarily represent those of their affiliated organizations, or those of the publisher, the editors and the reviewers. Any product that may be evaluated in this article or claim that may be made by its manufacturer is not guaranteed or endorsed by the publisher.

${ }^{\circ}$ Copyright: the Author(s), 2021

Licensee PAGEPress, Italy

Monaldi Archives for Chest Disease 2022; $92: 2103$

doi: 10.4081/monaldi.2021.2103

This article is distributed under the terms of the Creative Commons Attribution-NonCommercial International License (CC BY-NC 4.0) which permits any noncommercial use, distribution, and reproduction in any medium, provided the original author(s) and source are credited.

\section{Case Report}

A 52-year-old woman, never smoker, presented to Respiratory Intensive Care Unit (ICU) with a history of dyspnea on exertion for 6 months, progressed to dyspnea at rest over the last 15 days before admission along with non-productive cough for the past 3 months. There was no history of fever, hemoptysis, chest pain, pedal edema, syncope, loss of weight, or appetite. There was no environmental nor occupational exposure, symptoms of connective tissue disorder, or travel history. The patient had been taking bronchodilators (levosalbutamol and ipratropium bromide), a short course of antibiotics (azithromycin and piperacillin - tazobactam), and a course of prednisolone $30 \mathrm{mg}$ (tapered over 30 days) which did not bring any relief to her symptoms.

On presentation, her vital signs showed tachycardia (heart rate of 130 / $\mathrm{min}$, regular rhythm), normal blood pressure, tachypnoea (respiratory rate of $38 / \mathrm{min}$ with accessory muscles of respiration in use), afebrile and oxygen saturation of $70 \%$ on room air, $93 \%$ on non-rebreathing mask (NRBM). In view of the persistent respiratory distress, the patient was shifted to non-invasive ventilation (NIV) via an oronasal mask. On NIV at $\mathrm{FiO}_{2-}-70 \%$, positive end expiratory pressure (PEEP) of $10 \mathrm{~cm} \mathrm{H}_{2} \mathrm{O}$ and pressure support of 10 above PEEP, the arterial blood gas analysis revealed $\mathrm{pH} 7.34, \mathrm{pCO}_{2} 60, \mathrm{pO}_{2}$ $54, \mathrm{HCO}_{3} 24$ suggestive of respiratory acidosis and hypoxemia; $\mathrm{PaO}_{2} / \mathrm{FiO}_{2}$ ratio was 75 , suggestive of severe acute respiratory distress syndrome (ARDS). The general physical examination was essentially normal. Lung auscultation revealed inspiratory crackles in the bilateral infrascapular region. Cardiovascular and neuromuscular examinations were normal.

Routine laboratory evaluation showed normal complete blood count with differential count, renal function, and liver function tests. C reactive protein was positive, and NT pro-BNP was 850 $\mathrm{pg} / \mathrm{ml}$. Computed tomographic imaging demonstrated enlarged mediastinal lymph nodes, namely station 4R, 4L, 5, 7 (largest measuring $14 \mathrm{~mm}$ in short axis); bilateral diffuse areas of extensive ground-glass opacities with interlobular and intralobular septal thickening and nodular infiltrates, prominent involvement of upper lobes as compared to the lower lobes (Figure 1A). Differential diagnoses considered were sarcoidosis, chronic hypersensitivity pneumonitis and non-specific interstitial pneumonia. Subsequently, antinuclear antibody by indirect immunofluorescent assay was positive; extractable nuclear antigen antibody panel, rheumatoid factor, and anti cyclic citrullinated peptide were negative. Serum angiotensin-converting enzyme levels were normal.

In view of slightly elevated NT pro-BNP, patient was treated with parenteral furesemide $20 \mathrm{mg}$ twice a day for 2 days. The patient also demonstarted a favorable response to NIV with $\mathrm{P} / \mathrm{F}$ ratio improving to 170 within 24 hours of application. Once the $\mathrm{FIO}_{2}$ reduced to $40 \%$, the patient was given intermittent NIV for 2 hours alternating with non rebreathing mask for 2 hours. The patient 
underwent a flexible bronchoscopy with bronchoalveolar lavage (BAL), endobronchial biopsy (EBB), and transbronchial lung biopsy (TBLB) with NIV support on day 3 of admission. On inspection, vocal cords, trachea, and bronchial segments appeared normal. BAL fluid return was clear, and cytology showed few lymphocytes and alveolar macrophages. BAL fluid for AFB smear, gram stain, fungal stain were negative. BAL for Cartridge-based nucleic acid amplification test was positive for mycobacterium tuberculosis; with Rifampicin sensitive result. TBLB showed multiple well-formed necrotizing granulomas.

She was started on anti-tuberculosis treatment (ATT) with isoniazid (5 mg/kg), rifampicin (10 mg/kg), ethambutol $(15 \mathrm{mg} / \mathrm{kg})$ and pyrazinamide $(25 \mathrm{mg} / \mathrm{kg})$ as per National guidelines on the day 4 of admission on the basis of a positive CBNAAT report. The patient responded to treatment, with NIV weaned off in 6 days of admission, followed by gradually reduced oxygen requirement over 20 days, and was discharged on ATT. On follow-up in OPD after 4 months, dyspnea and cough had resolved. A repeat CT thorax demonstrated significant resolution of ground-glass opacities and nodular infiltrates (Figure 1B). The patient was advised to complete 6 months of ATT.

\section{Discussion}

The diagnosis of pulmonary tuberculosis in an intensive care unit provides challenges, including obtaining microbiological confirmation, and providing effective ATT in patients with poor absorption and high rates of organ dysfunction. Microbiological specimens can be obtained via endotracheal aspirate, non-directed bronchoalveolar lavage (miniBAL), or bronchoalveolar lavage via a flexible bronchoscope in mechanically ventilated patients. ET aspirate is easy to obtain, however, has lower specificity as compared to BAL. TBLB and BAL may increase the diagnostic yield but risk complications such as bleeding and pneumothorax, especially in a mechanically ventilated patient. FOB through a noninvasive ventilation mask facilitates sampling in a hypoxia non intimated patient. The sensitivity and specificity of CBNAAT in BAL are $80-86 \%$ and $90-93 \%$, respectively $[1,2]$.

The diagnostic yield for TBLB for chronic hypersensitivity pneumonitis ranges between $60-80 \%$ [3]. Similarly, there is high sensitivity of BAL + EBB + TBLB for sarcoidosis $(>80 \%)$. However, in the present case, the absence of cytopathological confirmation for inflammatory lung disorders and the presence of necrotizing granulomas suggestive of tuberculosis was surprising. The diagnosis of tuberculosis was further ascertained by a positive CBNAAT report which has a high sensitivity $(>97 \%)$ and specificity $(>90 \%)$ for diagnosis of tuberculosis in BAL $[4,5]$.

The presence of ANA is mainly associated with connective tissue disorders. The percentage of the population with falsepositive ANA varies between $2.5-15 \%$; reasons for which inlcude older age, viral infection, chronic tuberculosis, hypovitaminosis D, malignancy and drugs such as isoniazid and anticonvulsants [6].

Tuberculosis is an uncommon cause of severe hypoxemic respiratory failure. Despite being a public health problem of significant magnitude in India, the most extensive series of tuberculosis presenting with acute respiratory distress syndrome include only 18 cases over a long period of 16 years, with a reported mortality of $28 \%$ [7]. The reported mortality of tuberculosis associated with acute respiratory failure requiring mechanical ventilation varied from 50-80\% [8]. Bronchogenic pulmonary tuberculosis (TB) simulating a diffuse interstitial lung disease is a less recognized and rare cause of acute respiratory failure. The incidence of bronchogenic TB is reported between 1.5-1.9\%, with a $50-75 \%$ mortality rate in undiagnosed cases [9]. Causes of hypoxemia in non-
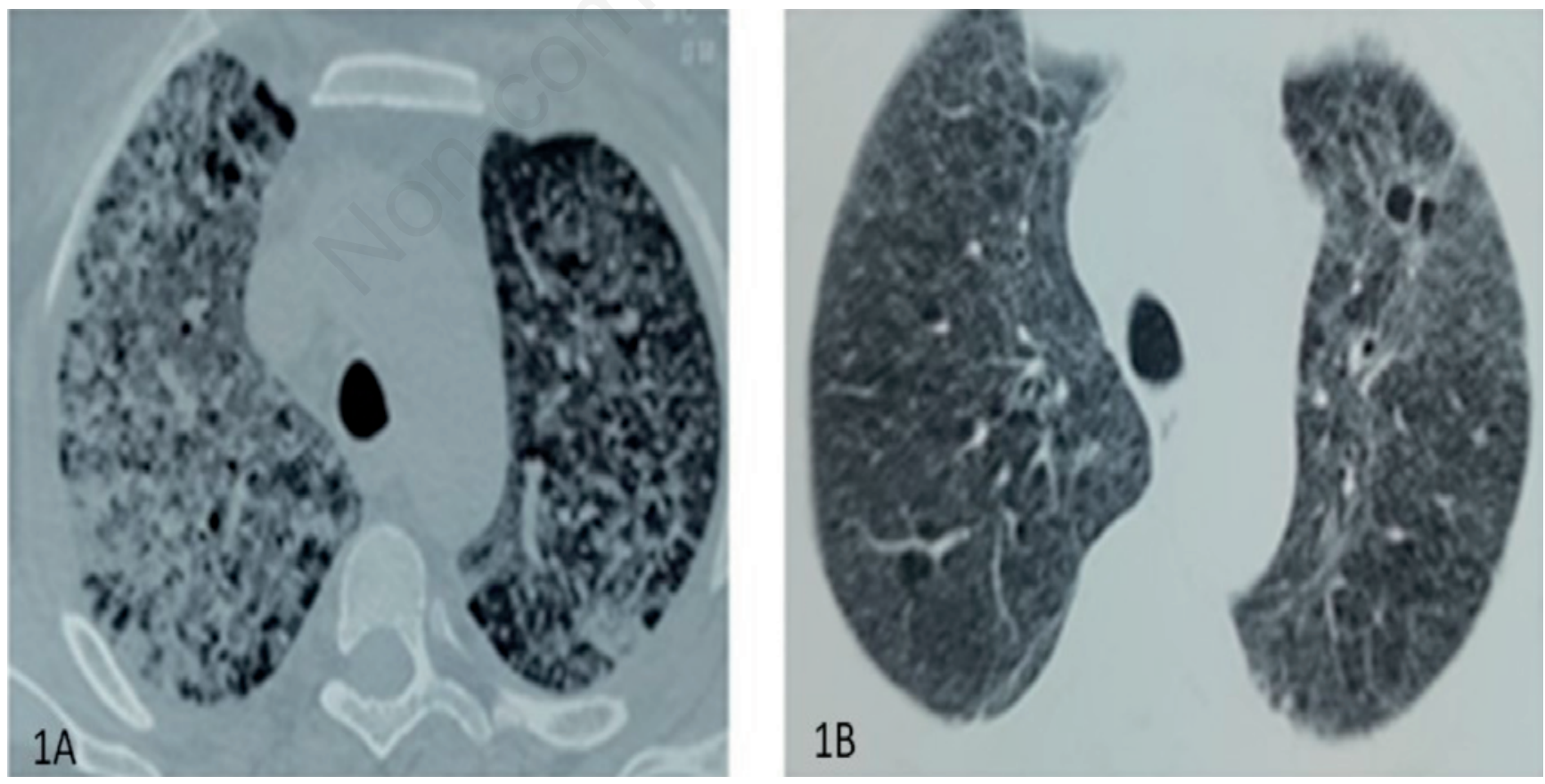

Figure 1. A) Representative axial lung window from the patient's initial chest computed tomographic scan demonstrates extensive ground-glass opacities with septal thickening and nodular infiltrates. B) Corresponding axial lung window from her subsequent CT scan obtained 4 months later shows marked resolution of ground-glass opacities and nodular infiltrates in bilateral lung fields. 
military pulmonary tuberculosis result from direct injury to alveolar epithelial cells from tubercular antigens. Lipoarabinomannan (LAM), a tubercular cell wall constituent, activates alveolar macrophages, triggering the production of TNF alpha, IL1 beta [10].

\section{Conclusions}

A thorough history, clinical examination, complete laboratory and radiological investigations and clinical correlation is necessary to ascertain the cause of respiratory failure. Bronchoscopy studies are instrumental in identifying an infective etiology when the cause of acute respiratory failure is uncertain. Tuberculosis should be considered as a differential diagnosis in unexplained respiratory failure and early diagnosis with appropriate treatment results in a favorable outcome.

\section{References}

1. Neves CP, Costa AG, Safe IP, et al. The role of minibronchoalveolar lavage fluid in the diagnosis of pulmonary tuberculosis in critically ill patients. BMC Infect Dis 2020;20:1-7.

2. Hagan G, Nathani N. Clinical review: Tuberculosis on the intensive care unit. Crit Care 2013;17:1-10.
3. Vasakova M, Morell F, Walsh S, et al. Hypersensitivity pneumonitis: Perspectives in diagnosis and management. Am J Respir Crit Care Med 2017;196:680-9.

4. Du Rand IA, Blaikley J, Booton R, et al. British Thoracic Society Bronchoscopy Guideline Group. British Thoracic Society guideline for diagnostic flexible bronchoscopy in adults: accredited by NICE. Thorax 2013;68:i1-i44.

5. Bashir YU, Nahvi N, Khan S, Jahan T. Diagnostic utility of bronchoalveolar lavage Xpert MTB/RIF assay in suspected cases of pulmonary tuberculosis. Int J Contemp Med Res 2019;6:F8-11.

6. Grygiel-Górniak B, Rogacka N, Puszczewicz M. Antinuclear antibodies in healthy people and non-rheumatic diseases diagnostic and clinical implications. Reumatologia 2018; 56:243-8.

7. Muthu V, Dhooria S, Aggarwal AN, et al. Acute respiratory distress syndrome due to tuberculosis in a respiratory icu over a 16-year period. Crit Care Med 2017;45:e1087-90.

8. Andresen M, Tapia P, Mercado M, et al. Catastrophic respiratory failure from tuberculosis pneumonia: Survival after prolonged extracorporeal membrane oxygenation support. Respir Med Case Rep 2013;10:19-22.

9. Kilaru SC, Prasad S, Kilaru H, et al. Active pulmonary tuberculosis presenting with acute respiratory failure. Respirol Case Rep 2019;7:1-5.

10. Dannenberg AM. Immune mechanisms in the pathogenesis of pulmonary tuberculosis. Rev Infect Dis 1989;11:S369-78. 